\title{
What gets filled-in during filling-in?
}

\author{
Frans W. Cornelissen and Tony Vladusich
}

Laboratory of Experimental Ophthalmology and BCN Neuroimaging Center, School of Behavioural and
Cognitive Neurosciences, University Medical Centre Groningen, University of Groningen, The
Netherlands. Correspondence to F.W.C. e-mail: f.w.cornelissen@rug.nl

In a recent review, Komatsu ${ }^{1}$ concluded that there is substantial evidence for neural mechanisms underlying the filling-in of visual properties such as contours, texture, brightness and colour. Komatsu ${ }^{1}$ stressed, however, that the evidence for filling-in depends on the details of the experiment and the type of filling-in phenomenon under investigation. We suggest here that recent human functional MRI (fMRI) studies provide a key to understanding what is and what is not filled-in during filling-in. In agreement with previous neurophysiological studies that failed to find evidence for neural surface filling-in ${ }^{2,3}$, these fMRI studies did not provide evidence that activity in the early visual cortex corresponds in a topographic (isomorphic) manner with illusory surface regions ${ }^{4,5}$. These recent findings conflict with some other fMRI reports in favour of isomorphic surface filling-in ${ }^{6,7}$ and with various studies of surface filling-in observed at the level of single neurons in monkey and cat visual cortices ${ }^{14}$.

How might these various findings be reconciled? Cornelissen et al. ${ }^{5}$ recently reported that fMRI activity elicited by luminance and colour edges is accurately modelled by assuming a linear combination of short-range $(\sim 1 \mathrm{~mm}$ along cortical surface) and long-range $(>15 \mathrm{~mm})$ responses (FIG. 1a,b). Both short- and long-range responses are symmetrical (Gaussian) with respect to the inducing edge and therefore neither corresponds topographically with surface brightness and colour. The long-range component of these responses $\square$ which extend up to 5 degrees away from the stimulus edge, similar to the non-classical receptive field properties of single neurons $\square$ calls into question the validity of positive fMRI and neurophysiological reports of cortical surface filling-in. Stated plainly, many positive reports of surface filling-in can be understood as an artefact of long-range cortical responses to edge stimuli. The fMRI activity maps of Sasaki and Watanabe ${ }^{6}$, for example, are qualitatively more consistent with long-range edge-centred responses than uniformly filled-in surface responses (FIG.1c). Recent modelling of the responses of single neurons of the primary visual cortex to stimuli that induce illusory brightness contrast ${ }^{8}$ supports the notion that long-range cortical responses are unrelated to surface filling-in in most surface responsive neurons.

Future studies of filling-in also need to distinguish between the filling-in of contour and surface information. For example, Meng et al. ${ }^{7}$ showed that the perception of illusory visual phantoms is correlated with fMRI activity in the primary visual cortex. The authors $\square$ stimuli induce the perception of strong illusory contours, along with a weaker surface filling-in effect. Although we do not question the evidence for the completion of illusory contours in the visual cortex, we do emphasize that the case in favour of cortical surface fillingin is weakened by recent findings.

1. Komatsu, $\mathrm{H}$. The neural mechanisms of perceptual filling -in. Nature Rev. Neurosci. 7,

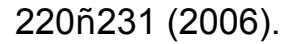

2. Friedman, H. S., Zhou, H. \& von der Heydt, R. The coding of uniform colour figures in monkey visual cortex. J. Physiol. 548, 593613 (2003).

3. von der Heydt, R., Friedman, H. S. \& Zhou, H. in Filling-in: From Perceptual

Completion to Skill Learning (eds Pessoa, L. \& De Weerd, P.) $106 \square 127$ (Oxford Univ. Press, New York, 2003).

4. Perna, A., Tosetti, M., Montanaro, D. \& Morrone, M. C. Neuronal mechanisms for illusory brightness perception in humans. Neuron 47, 645651 (2005).

5. $\quad$ Cornelissen, F. W., Wade, A. R., Vladusich, T., Dougherty, R. F. \& Wandell, B. No functional magnetic resonance imaging evidence for brightness and colour filling -in in early

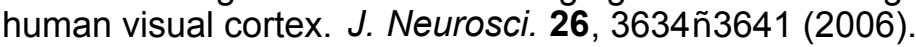

6. Sasaki, Y. \& Watanabe, T. The primary visual cortex fills in color. Proc. Natl Acad. Sci. USA 101, $18251 \square 18256$ (2004).

7. Meng, M., Remus, D. A. \& Tong, F. Filling -in of visual phantoms in the human brain. Nature Neurosci. 8, $1248 \square 1254$ (2005).

8. Kinoshita, M. \& Komatsu, H. Neural representation of the luminance and brightness of a uniform surface in the macaque primary visual cortex. J. Neurophysiol. 86, 25592570 (2001). 
9. Rossi, A. F., Rittenhouse, C. D. \& Paradiso, M. A. The representation of brightness in primary visual cortex. Science 273, $1104 \square 1107$ (1996).

10. Rossi, A. F. \& Paradiso, M. A. Neural correlates of perceived brightness in the retina, lateral geniculate nucleus, and striate cortex. J. Neurosci. 19, 61456156 (1999).

11. Peng, X. \& Van Essen, D. C. Peaked encoding of relative luminance in macaque areas V1 and V2. J. Neurophysiol. 93, $1620 \square 1632$ (2005).

12. MacEvoy, S. P. \& Paradiso, M. A. Lightness constancy in primary visual cortex. Proc. Natl Acad. Sci. USA 98, 88278831 (2001).

13. Hung, C. P., Ramsden, B. M., Chen, L. M. \& Roe, A. W. Building surfaces from borders in Areas 17 and 18 of the cat. Vision Res. 41, $1389 \square 1407$ (2001).

14. Roe, A. W., Lu, H. D. \& Hung, C. P. Cortical processing of a bri ghtness illusion. Proc. Natl Acad. Sci. USA 102, 38693874 (2005).

15. Vladusich, T., Lucassen, M. P. \& Cornelissen, F. W. Do cortical neurons process luminance or contrast to encode surface properties? J. Neurophysiol 95, $2638[2649$ (2006).

Acknowledgements:

F.W.C. and T.V. are supported by a grant from the Cognition Program of the Netherlands Organization for Scientific Research (NWO) and the School of Behavioural and Cognitive Neurosciences.

Figure 1 | Long-range symmetrical responses to edge stimuli may explain previous reports of cortical surfacefilling-in. a I fMRI activity in the primary visual cortex during brightness changes elicited in disc-surround stimuli by either luminance modulation of the central disc (open circles) or induced by modulating the surround (filled circles). Responses were strongest at the transition between the central disc and surround (0 mm), and decreased with increasing distance from this edge. b | Responses could be accurately modelled with a linear combinationof short- and long-range Gaussian edge responses and a small luminance response (see inset). The long-range edge response extends over several millimetres of cortical surface (full-width half-maximum > $15 \mathrm{~mm}$ ) and is symmetrical around the edge. Model results are shown for luminance modulation of the central disc. c I Cortical responses to stimuli eliciting neon colour spreading. Note that responses are evidently strongest near illusory edges (dotted lines indicated by white arrows) and decrease with distance from the edge. We suggest that such responses are better understood in terms of lonerange Gaussian responses than uniform filled-in surface responses. Panels a and b reproduced, with permission, fromREF.5 (C) (2006) Society for Neuroscience. Panel c reproduced, with permission, from REF.6 (C) (2004) National Academy of Sciences. 